observed in the sublining inflammatory cells including plasma cells (CD38+) and macrophages (CD68+), mainly located in the cytoplasm. (C) Detection of HBV S gene in RA synovium by nested PCR. Nested PCR showed HBV S gene only in all four CHB synovial tissues. Liver tissue from a patient with HBV-related hepatocellular carcinoma was used as positive control.

Conclusions: Our results reveal definite presence of HBV in the synovium which may be involved in the pathogenesis of local lesion and exacerbate disease progression in RA patients with $\mathrm{CHB}$.

Acknowledgements: This work was supported by National Natural Science Foundation of China (grant No. 81671612 and 81601427), Guangdong Natural Science Foundation (grant No. 2016A030313307 and 2016A030313307) and Fundamental Research Funds for the Central Universities (grant No. 17ykjc12). Disclosure of Interest: None declared DOI: 10.1136/annrheumdis-2018-eular.6072

\section{SAT0148 ORAL CARE AND HEALTH IN RHEUMATOID ARTHRITIS PATIENTS BASED ON A SELF- ASSESSMENT QUESTIONNAIRE INVESTIGATION}

L. L. Strandbygaard ${ }^{1}$, M. Radwan-Oczko ${ }^{2}$, I. Dus ${ }^{2}$, A. M. Thomsen ${ }^{1}$, K. Schmøkel ${ }^{3}$, C. Rasmussen 1. 'Department of Rheumatology, North Jutland Regional Hospital, Hjørring, Hjørring, Denmark, ${ }^{2}$ Department of Oral Pathology, Wroclaw Medical University, Wroclaw, Poland, ${ }^{3}$ Municipal Pediatric Dental Care \& Dental Care for the Impaired, Hjørring, Denmark

Background: Some investigations indicate a higher prevalence of periodontal disease (PD) in rheumatoid arthritis (RA) patients compared to healthy individuals without RA. Significant improvements in clinical parameters and laboratory tests were demonstrated in RA patients following periodontal therapy. Periodontal diseases - gingivitis and periodontitis are inflammatory, multifactorial pathologies of periodontal tissues, which support the teeth. The factor responsible for the inflammation is a plaque with specific bacteria.

In gingivitis, which is the first stage of PD, only gingiva is involved. Then in susceptible patients, periodontitis is developing and the deeper laying structures surrounding the teeth are involved and destroyed. The patients' own observation of gingival bleeding strongly indicates gingival disease.

The effectiveness of oral hygiene, including self-care, in controlling periodontal health is crucial and supported by several studies. RA patients frequently experience reduced function of fingers and wrists that makes oral hygiene troublesome. Objectives: The purpose of this study was to asses RA patient's oral care and health based on a self-assessment questionnaire.

Methods: Questionnaires were mailed to 300 patients treated for RA in a Danish rheumatology outpatient clinic.

Results: A total of 164 patients completed the questionnaires. The mean age of patients was 65 years, and the average value of DAS28 was 2,31 . Twelve percent were active smokers. The "dry mouth" syndrome, as a problem when chewing or swallowing, pointed out $4 \%$ of patients. Difficulties in biting or chewing revealed $10 \%$ of patients. As much as $87 \%$ stated they regularly visit a dentist, min. one time a year.

Regarding self-oral care $15 \%$ of patients answered, they brush teeth only once a day. Most of the patients (51\%) used manual toothbrush vs electrical - which can be more convenient for patients with problems of the hands/wrists. Only $21 \%$ used mouth-rinsing liquid. Difficulties in performing home oral hygiene procedures were reported by $9 \%$ when brushing teeth, and by $17 \%$ using dental floss or interdental brushes. $11 \%$ of responders rated the status of their gingiva as poor. Gingival bleeding spontaneous or related to brushing was experienced by respectively $15 \%$ and $49 \%$ of patients. No patients stated knowledge of an association between oral health and RA.

Conclusions: The oral care in RA patients, including self-care, seems to require improvement. Providing important information to the patients about the relationship between the oral/periodontal health and RA disease activity should raise the patient's awareness, which may improve the course of the RA disease.

\section{REFERENCES :}

[1] Choi IA, et al. Periodontitis is associated with rheumatoid arthritis: a study with longstanding rheumatoid arthritis patients in Korea.

[2] Fuggle NR, et al. Hand to mouth: a systemic review and meta-analysis of the association between rheumatoid arthritis and periodontitis.

Disclosure of Interest: None declared

DOI: 10.1136/annrheumdis-2018-eular.4772

\section{SAT0149 IS ESTABLISHED SERONEGATIVE RHEUMATOID ARTHRITIS EVEN A MILD FORM OF THE DISEASE?}

L. D. Fajardo Hermosillo ${ }^{1} .{ }^{1}$ Rheumatology. Internal Medicine Division, IMSS HGR110, Guadalajara, Mexico

Background: Established Seronegative Rheumatoid Arthritis (SNRA) was considered a mild form of the disease with a good response to therapy on the past ${ }^{1}$. Lately evidence indicates this form of arthritis in early stage is serious and should not be underestimated in terms of disease activity and radiographic damage ${ }^{2}$. By other hand early SNRA presents shorter symptoms duration and different response to therapy compare to seropositive (SP) patients ${ }^{3,4}$. At present, the influence of seronegative (SN) status of clinical course and treatment choice is still controversial. Data of this issue are scarce and often insufficiently powered.

Objectives: To determine demographical, clinical and treatment differences between established SN and SP Rheumatoid Arthritis (RA) in a Mexican cohort. Methods: 511 patients with established RA ( $>2$ years from time at onset of diag nosis) that fulfilled ACR/EULAR 2010 criteria ( $\geq 18$ years) from a Mexican cohort from 2012 to 2017 were examined. Patients without presence of rheumatoid factor (RF) and anti-citrullinated protein antibodies (ACPA) were considered SN. Demographic factors, clinical features, disease activity measured using DAS28, functional status, comorbidities and pharmacologic treatments were examined for patients with established SN and SP RA. Charlson's clinical co-morbidity index was used to analyze comorbidities. Chi-square and Student-t test was performed by univariate analysis and logistic regression was used by multivariate analysis, both were adjusted for age and gender. Standard deviation and mean of modified Charlson's index was obtained by ANOVA analysis.Statistical test were conducted at $5 \%$ level of significance.

Results: Of 511 patients with established RA $89 \%$ were women. The mean age [standard deviation (SD)] was $55.6(11.8)$ years. The mean of time at onset of RA (SD) was 12.1 (8.7) years. A total of 77 (15.1\%) patients had established SNRA. In the univariable analyses established SNRA patients were more likely to have shorter time at onset of RA, minor disease activity and radiographic progression, lesser extra-articular and sicca manifestations, reduced demand of hip and knee arthroplasty, also present lower comorbidities including osteoporosis and fibromyalgia, besides to use fewer methotrexate and corticosteroids. As expected, the modified Charlson's comorbidity index score was lower in established SNRA patients. No differences were found for use of biologic agents in both groups. In multivariable analyses, minor disease activity (OR $0.33,95 \% \mathrm{Cl} 0.17-0.60$, $\mathrm{p} \leq 0.001$ ), less frequently use of methotrexate (OR $0.38,95 \% \mathrm{Cl} 0.17-0.82$ $\mathrm{p}=0.014$ ) and minor radiographic progression (OR $0.13,95 \% \mathrm{Cl} 0.40-0.39$, $\mathrm{p} \leq 0.001$ ), remained significant in established SNRA patients.

Conclusions: This study suggests that established SNRA present less disease activity and radiographic damage as well as fewer use of MTX than established SPRA. However differences in activity disease and response to treatment can be found in early stages of RA not included in this study. Therefore early SNRA cannot be considered to be a generally mild form of the disease like as established SNRA. These observations must be confirmed in larger studies including early stages of RA.

\section{REFERENCES}

[1] Nat Rev Rheumatol 2012;8:144-152.

[2] J Rheumatol 2014;41;2361-2369.

[3] Ann Rheum Dis 2012;71:245-248.

[4] Arthritis Res Ther 2014;16: R13.

Disclosure of Interest: None declared

DOI: 10.1136/annrheumdis-2018-eular.6135

\section{SAT0150 INADEQUATE CARDIOVASCULAR RISK MANAGEMENT IS NOT RHEUMATOID ARTHRITIS SPECIFIC AMONG PATIENTS WITH PREVALENT RHEUMATIC AND MUSCULOSKELETAL DISEASES}

M. Reyskens ${ }^{1}$, L. Tsang ${ }^{1}$, M. Walschot ${ }^{1}$, F. Fleurinck ${ }^{1}$, A. O. Solomon ${ }^{2}$, A. Millen ${ }^{2}$,

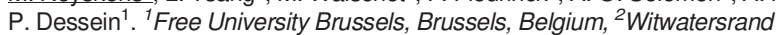
University, Johannesburg, South Africa

Background: Preventive pharmacotherapy for atherosclerotic cardiovascular disease (ACVD) is reportedly underused in patients with rheumatoid arthritis (RA) Whether this shortcoming is RA specific amongst patients with prevalent rheumatic and musculoskeletal diseases (RMD) is currently unknown.

Objectives: This study aimed to compare high ACVD risk profiles and statin use by indication between RA and non-RA patients with RMD

Methods: We investigated 470 consecutive RMD patients of which 80 had RA 92 undifferentiated inflammatory/early arthritis (UIA), 127 fibromyalgia and 171 